\title{
Prospective evaluation of the renal morphology and vascular resistance in patients with ankylosing spondylitis.
}

\author{
Mustafa Resorlu¹, Ferhat Gokmen², Hatice Resorlu², Gurhan Adam¹, Nilufer Aylanc1, \\ Ayla Akbal' ${ }^{2}$ Huseyin Ozdemir ${ }^{1}$
}

${ }^{1}$ Department of Radiology, ${ }^{1}$ Department of Physical Medicine and Rehabilitation,Canakkale Onsekiz Mart University, Faculty of Medicine, Canakkale, Turkey

\begin{abstract}
Aims: To evaluate renal morphology, prevalence of urinary stone disease, renal perfusion and resistance to renal blood flow in patients with ankylosing spondylitis(AS). Material and methods: Thirty-eight patients diagnosed with AS and with normal basal renal functions, together with 38 healthy individuals matched in terms of age, sex, blood lipid profile and body mass index, were included. Total cholesterol, triglyceride, urea, creatinine and glucose levels were measured in both groups, as well as vitamin D, erythrocyte sedimentation rate (ESR) and C-reactive protein in the AS group. Renal dimensions, parenchymal echogenicity, presence of stone and renal resistive index (RRI) from the interlobular artery level were measured, and correlations with clinical and laboratory parameters were assessed. Results: Thirty-eight patients diagnosed with AS (age $42.4 \pm$

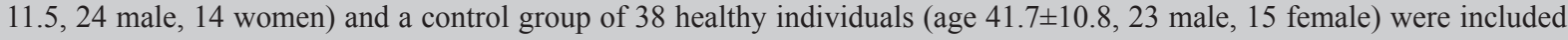
in the study. Renal stone was present in 7 patients (18.4\%) in the AS group and 4 subjects (10.5\%) in the control group. There was no significant difference in prevalence of stone between the groups $(\mathrm{p}=0.516)$. RRI values were significantly higher in the patients with AS $(0.63 \pm 0.06)$ compared with the control group $(0.59 \pm 0.03, p=0.001)$. Significant correlations were determined between RRI and age, triglyceride level, body mass index and length of disease. Conclusions: Renal Doppler is an important examination in early diagnosis and monitoring of renal changes in AS patients since renal complications in AS develop in the chronic and follow a subclinical course.
\end{abstract}

Keywords: ankylosing spondylitis,ultrasound,renal morphology, renal vascular resistance

\section{Introduction}

Ankylosing spondylitis (AS) is a chronic inflammatory disease of uncertain etiology, a member of the seronegative spondyloarthropathies, primarily involving the axial skeleton and sacroiliac joints [1]. The disease is generally diagnosed in the third decade and is more common in males [2]. Since AS is a chronic disease, with systematic inflammatory process, it can also lead to the involvement of different organs. Involvement of organs

Received 25.11.2014 Accepted 25.01.2015

Med Ultrason

2015, Vol. 17, No 2, 180-184

Corresponding author: Mustafa Resorlu, MD

Canakkale Onsekiz Mart Universitesi,

Terzioglu Yerleskesi, Barbaros Mh, 17100,

Canakkale, Turkey

Phone/Fax:+90 505454 8722/+90 2862180393

E-mail: mustafaresorlu77@gmail.com such as the heart, lung, eye, bone, and in particular kidney has a severe effect on quality of life and mortality $[2,3]$. Immobility, inflammatory cytokines, long-term non-steroid anti-inflammatory drug (NSAID) use and changes taking place in bone turnover lead to irregularities in calcium metabolism in AS, and these to the formation of stone in the urinary system [4]. However, the results from the few studies concerning the prevalence of urolithiasis are inconsistent $[1,4]$.

Apart from stone disease in these patients, long-term NSAID use, amyloid deposition in the kidneys, an increased incidence of glomerulonephritis, a rise in immunoglobulin A nephropathy and atherosclerosis can also affect renal functions [5]. Non-invasive techniques such as gray-scale ultrasonography (US) and Doppler US are often preferred for evaluation of the patients being easily performed and repeatable techniques. Kidney dimensions, cortical thickness and collecting system dilation and pa- 
renchymal echogenicity can be assessed using gray-scale US. Renal resistivity index (RRI) can be measured with colorDoppler US, and renovascular and renal parenchymal diseases can be monitored using changed in the wave form of the intrarenal arteries [6-8]. The purpose of this study was to evaluate kidney morphology, prevalence of urinary stone, resistance to blood flow and renal perfusion using US in patients with AS without urinary symptoms and with normal basal functions.

\section{Material and methods}

\section{Patient Population}

Thirty-eight patients diagnosed with AS on the basis of ASAS diagnostic criteria and applying to the Canakkale Onsekiz Mart University, Faculty of Medicine, Department of Physical Medicine and Rehabilitation (age $42.4 \pm 11.5$ years, 24 male, 14 female) were included in the study following ethical committee approval. Written informed consent was obtained from each patient. Sixteen patients were using NSAID drugs and 12 were using sulfasalazine. Subjects with diabetes, hypertension, hyperlipidemia, proteinuria, hematuria, pyuria, renal insufficiency, other inflammatory rheumatic disease or connective tissue disease, malignity, active infectious disease, vasculitis, or hepatic parenchymal disease based on medical history, physical examination or laboratory results were excluded. Subjects with history of myocardial infarction or peripheral arterial disease, patients using cigarettes or alcohol and patients on TNF- $\alpha$ blockers treatment were also excluded. The control group was selected among healthy individuals with no cardiovascular or metabolic disease. Thirty-eight age-, sex-, blood lipid profile- and body mass index-matched healthy individuals (41.7 \pm 10.8 years, 23 male, 15 female) were enrolled as the control group.

Heavy exercise and alcohol use were prohibited to all patients prior to laboratory tests, and blood specimens were collected after $12-\mathrm{h}$ fasting. Total cholesterol, triglyceride, urea, creatinine and glucose levels were measured in both groups, as well as vitamin D, erythrocyte sedimentation rate (ESR) and C-reactive protein levels in the AS group. Bath Ankylosing Spondylitis Activity Index (BASDAI) and Bath Ankylosing Spondylitis Functional Index (BASFI) values were determined for the patient group. Blood pressure was measured from both arms using a sphygmomanometer with the patient in a seated position. BMI was calculated as the ratio of weight to height squared $\left(\mathrm{kg} / \mathrm{m}^{2}\right)$.

\section{Ultrasound Protocol and Image Analysis}

NSAID intake was stopped $24 \mathrm{~h}$ before RDUS examinations and food intake $10 \mathrm{~h}$ before so that these did not affect the test results. All US examinations were performed by the same blinded radiologist. Gray-scale US and color Doppler US were performed with a renal protocol using a Toshiba Aplio XG Doppler US device and a convex transducer (PVT-375BT). Renal long axis, parenchymal thickness, presence of stone and resistive index echogenicity and interlobular level were measured in both kidneys. Peak systolic velocities were measured in the abdominal aorta and renal arteries, and patients with a renoaortic rate $>3.5$ or a peak systolic velocity $>$ $125 \mathrm{~cm} / \mathrm{s}$ in any segment were excluded from the study. Parenchymal thickness measurements were performed based on the shortest distance between the renal sinus and the capsule in the superior pole, interpolar region and inferior pole, and mean values were calculated. Renal parenchymal echogenicity was compared with hepatic echogenicity only in patients with no hepatosteatosis. In patients with hepatosteatosis, renal parenchyma was assessed by comparing with splenic echogenicity. Doppler US examinations were performed on the level of the interlobular arteries with the angle of $30^{\circ}$ to $60^{\circ}$, and at the lowest level of pulse repetition frequency (PRF). RRI values were determined with the mean of three separate measurements in the renal superior pole, interpolar regional and inferior pole. Renal stones were defined as hyperechoic spots with acoustic shadow.

\section{Statistical Analysis}

Analysis of the data obtained was performed on SPSS 16.0 Windows software. The statistical test to be applied was decided on using the Shapiro-Wilk normality test. The Independent Samples T test was used to compare means between the two groups, while the chi square and Fisher's exact test were used to analyze categoric variables. Correlation between constant variables in the two groups was investigated using Spearman's and Pearson's correlation tests. The Mann Whitney U test was used to assess non-normally distributed means. Significance was set at a $\mathrm{p}$ value below 0.05 .

\section{Results}

Thirty-eight patients with AS (age $42.47 \pm 11.57$ years) and a control group of 38 healthy individuals (age $41.73 \pm 10.87$ years) were enrolled. The control group consisted of 15 women and 23 men, and the AS group of 14 women and 24 men. There was no significant difference between the groups in terms of age or sex $(p=0.776$ and $\mathrm{p}=0.813$, respectively). No significant difference was also observed between the patients with $\mathrm{AS}$ and the control group in terms of total cholesterol, triglyceride, blood urea or creatinine levels. Demographic data and laboratory results for both groups are shown in Table I. 
Table I. Patient and control group demographic characteristics and laboratory values

\begin{tabular}{llll}
\hline & $\begin{array}{l}\text { Control Group } \\
(\mathbf{n = 3 8})\end{array}$ & $\begin{array}{l}\text { Ankylosing Spondylitis } \\
(\mathbf{n = 3 8})\end{array}$ & p value \\
\hline Mean Age ( \pm SD) & $41.7 \pm 10.8$ & $42.4 \pm 11.5$ & 0.776 \\
Triglyceride (mg/dL) & $96.9 \pm 29.1$ & $87.5 \pm 27.1$ & 0.156 \\
Urea (mg/dL) & $26.9 \pm 4.8$ & $28.6 \pm 8.4$ & 0.296 \\
Creatinine & $0.74 \pm 0.11$ & $0.77 \pm 0.13$ & 0.242 \\
Renal dimension (mm) & $109 \pm 7.5$ & $107.9 \pm 10.7$ & 0.614 \\
Parenchymal thickness (mm) & $12.6 \pm 2.2$ & $13.05 \pm 1.9$ & 0.449 \\
Urinary stone disease & $10.5 \%$ & $18.4 \%$ & 0.516 \\
RRI & $0.59 \pm 0.03$ & $0.63 \pm 0.06$ & $\mathbf{0 . 0 0 1}$ \\
BMI (kg/m2) & $26.3 \pm 3.8$ & $27.9 \pm 6.09$ & 0.191 \\
ESR (mm/h) & - & $22.4 \pm 15.8$ & - \\
\hline
\end{tabular}

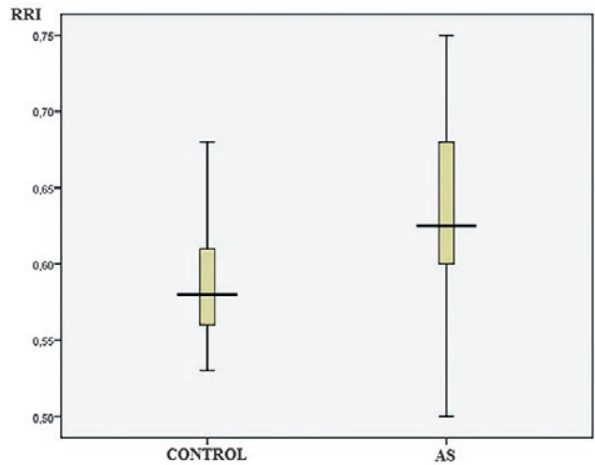

Fig 1. Comparison of RRI value between ankylosing spondylitis patients and control cases

Mean duration of disease in the AS group was $13.13 \pm 8.77$ years, BASFI score $3.16 \pm 1.58$, and BASDAI score $2.83 \pm 1.19$. Renal long axes in the patient and control groups were $10.79 \pm 1.07 \mathrm{~cm}$ and $10.90 \pm 0.75 \mathrm{~cm}$, respectively; and renal parenchymal thickness was $13.05 \pm 1.98$ $\mathrm{mm}$ and $12.68 \pm 2.23 \mathrm{~mm}$ in the patient and control groups, respectively. There was no difference between the groups in terms of renal long axis or renal parenchymal thickness $(\mathrm{p}=0.614, \mathrm{p}=0.449$, respectively). Renal stone was present in 7 patients $(18.4 \%)$ in the AS patient group and 4 subjects $(10.5 \%)$ in the control group. There was

Table II. Correlation of study parameters with RRI in patients with ankylosing spondylitis

\begin{tabular}{lll}
\hline Parameters & r & p value \\
\hline Age (years) & 0.685 & $<\mathbf{0 . 0 0 1}$ \\
Duration of disease (years) & 0.587 & $<\mathbf{0 . 0 0 1}$ \\
BMI $(\mathrm{kg} / \mathrm{m} 2)$ & 0.501 & $\mathbf{0 . 0 0 1}$ \\
Triglyceride $(\mathrm{mg} / \mathrm{dL})$ & 0.385 & $\mathbf{0 . 0 1 7}$ \\
Creatinine $(\mathrm{mg} / \mathrm{dL})$ & 0.065 & 0.699 \\
Cholesterol $(\mathrm{mg} / \mathrm{dL})$ & 0.167 & 0.317 \\
BASFI & -0.022 & 0.896 \\
BASDAI & -0.212 & 0.202 \\
ESR $(\mathrm{mm} / \mathrm{h})$ & 0.125 & 0.453 \\
Glucose $(\mathrm{mg} / \mathrm{dL})$ & 0.264 & 0.110 \\
\hline
\end{tabular}

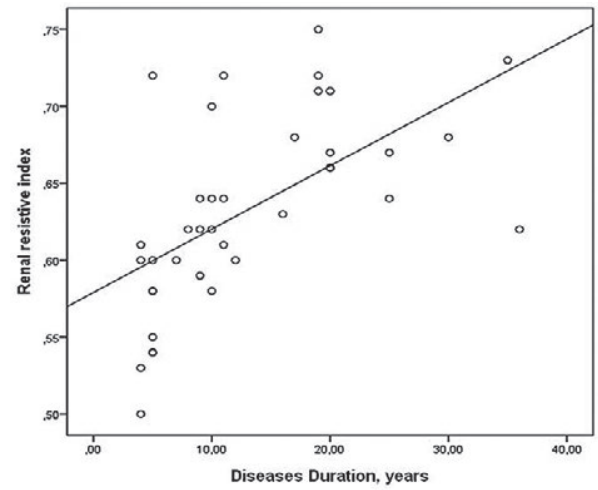

Fig 2. Association between RRI value and disease duration in patients with ankylosing spondylitis

no significant difference in terms of prevalence of renal stone between the two groups $(\mathrm{p}=0.516)$. RRI value in the AS patient group $(0.63 \pm 0.06)$ was significantly higher than that in the control group $(0.59 \pm 0.03)(\mathrm{p}=0.001$, fig 1). Correlations between RRI value and age, triglyceride levels, BMI and duration of disease were significant (fig 2). No significant correlations were determined between RRI and urea, creatinine, total cholesterol, vitamin D and blood glucose levels and BASFI or BASDAI. The relations between RRI and other parameters in the AS patient group are shown in table II.

\section{Discussions}

AS is an inflammatory rheumatic disease particularly affecting the spine and sacroiliac joints. It is also capable of giving extra-articular clinical findings [9]. Symptoms frequently start in early adulthood. The most common non-skeletal finding is acute anterior uveitis or iridocyclitis [10].The more rarely seen cardiac and pulmonary involvement affects mortality. In addition to increased carotid intima media thickness and epicardial fat tissue, ascending aortitis, aortic valve insufficiency and transmission defects may also be observed [11,12]. Airway 
disease, interstitial anomalies and pleural thickening occur in pulmonary involvement [13].

Although renal complications in AS are well known and have been studied in detail, there are limited studies of urolithiasis in these patients and inconsistent results have been obtained $[1,4]$. Calcium metabolism in AS is impaired by spinal immobility, released inflammatory cytokines, new bone formations and prolonged NSAID use, and this constitutes a risk factor for urinary stone disease [4]. Korkmaz et al reported a higher prevalence of renal stone in patients with AS (20\%) compared to patients with Behcet's disease (5.5\%) and healthy individuals (3.3\%). They also reported that patients with renal stones had a longer duration time of AS disease [1]. Similarly, Canales et al [14] determined an increased prevalence of urolithiasis in patients with spondylarthritis but İncel et al [4] reported no difference in prevalence of urolithiasis. In our study, renal stone was present in 7 patients with AS $(18.4 \%)$ and in 4 subjects in the control group (10.5 $\%)$ but without statistical significance $(p=0.516)$. Since $80-90 \%$ of urinary stones are calcium-based, and due to impair of calcium metabolism in AS, our hypothesis was that there would be a greater prevalence of urinary stone in the disease. We think that the limited size of our patient group represents a limitation and that wider-ranging studies are now needed.

Renal disorders without stonesare also on the increase in patients with AS (due to glomerulonephritis, renal amyloid deposition, prolonged NSAID use, uncontrollable systemic inflammation, and atherosclerosis) [15]. Rheumatic diseases, particularly rheumatoid arthritis and AS, are regarded as major causes of reactive amyloidosis. Clinically apparent amyloidosis is relatively rare in studies of the prevalence of amyloidosis in patients with AS. Dönmez S et al determined a prevalence of clinically apparent amyloidosis of 1.1\% [16]. In studies investigating subclinical amyloidosis by analyzing the abdominal fat pad, Gratacos et al identified amyloidosis in 7\% of patients and Sing $\mathrm{Y}$ et al in $6.9 \%$ [5,17]. NSAIDs exhibit their effects by inhibiting the enzymes cyclo-oxygenase 1 (COX-1) and cyclooxygenase $2(\mathrm{COX}-2)$ that play a role in the synthesis of prostaglandin. COX-1 is responsible for renal vascular dilation and decreased renal vascular resistance, and thus for an increase in renal perfusion [18]. Chronic interstitial nephritis or renal papillary necrosis can develop in analgesic nephropathy. Similarly, acute kidney failure, interstitial nephritis, nephrotic syndrome, decreased blood flow in the inner cortical nephrons, water and salt retention, hyporeninemic hypoaldosteronism and hypercalcemia may develop secondary to vasoconstriction with NSAIDs [19].
Platt et al showed that RRI increase in patients with reno-vascular and interstitial renal disease, and they also reported an increase of RRI in patients with lupus nephritis in another study $[20,21]$. In another study, Aikimbaev et al showed an increase in RRI on progressive systemic sclerosis with renal involvement [22]).

One important finding from this study is that RRI values in patients with AS were significantly higher compared with those of the control group. Since there are no other comparable studies in the literature we were unable to compare our results, although bearing in mind that complications cited above that may also follow a subclinical course in AS, this was an expected finding. Similarly, we think that increased subclinical atherosclerotic changes in AS also affect the rise in RRI. Alterini et al determined a correlation between an increase in CIMT, endothelial dysfunction and left ventricular hypertrophy and an increase in RRI values [23]. We think that the correlation between RRI values and triglyceride levels and BMI in our study may also be associated with the relation between these two parameters and atherosclerosis.

Another important finding from this study is the rise in RRI values in parallel with the duration of disease and age. This is important due to renal complications in AS being time-dependent and progressive. Mean duration of disease in this study was 13.1 years, a sufficient period for renal complications to arise and progress. Amyloidosis has been reported to be positively correlated with patient age and duration of disease in some studies [16,17]. There are also recent studies showing a positive correlation between patient age and duration of disease and atherosclerosis leading to the compromise of the renal perfusion and renovascular pathology [24].

BASFI and BASDAI were also correlated with duration of disease or RRI in this study. BASFI and BASDAI show the periodic functionality and activity of the disease at the time. Both indices exhibiting a correlation with increased RRI as a result of a progressive process is also an expected finding.

This is a prospective study from a single institution with several limitations. First, results are based on a relatively small sample size; therefore, these findings must be confirmed by further large prospective studies. Second, we did not use the pulsatility index in this study, correlations with RI could be important. Finally, the most important limitation of the present study was that subjects with proteinuria, hematuria or pyuria on urine analyze were excluded from study. Therefore, we could not perform a correlation between RI and the presence of hematuria and/or proteinuria as markers of parenchimal disease in AS. Despite these shortcomings, this is an important study, because there is no data in the literature 
that evaluates kidney morphology, prevalence of urinary stone, resistance to blood flow and renal perfusion using US in patients with AS.

\section{Conclusions}

Renal involvement in AS is a chronic process and changes taking place secondary to NSAID use, analgesic nephropathy and atherosclerosis, increase the importance of early diagnosis and monitoring of these subclinical courses of processes such as amyloidosis. We think that RRI, used in the monitoring of renovascular and renal diseases, can also be used as a predictor of renal complications in patients with AS. Further studies are now required on the subject.

\section{Conflict of interest: none}

\section{References}

1. Korkmaz C, Ozcan A, Akcar N. Increased frequency of ultrasonographic findingssuggestive of renal stones in patients with ankylosing spondylitis. Clin Exp Rheumatol 2005; 23: 389-92

2. Elewaut D, Matucci-Cerinic M. Treatment of ankylosing spondylitis and extra-articular manifestations in everyday rheumatology practice. Rheumatology 2009; 48: 10291035.

3. Lange U, Stapfer G, Ditting T, et al. Pathologic alterations of the heart and the kidney in patients with ankylosing spondilitis. Eur J Med Res 2007; 12: 573-581.

4. Incel NA, Gökoğlu F, Nacir B, Incel N. Bone and stone in ankylosing spondylitis: osteoporosis and urolithiasis. Clin Rheumatol 2006; 25: 667-670.

5. Gratacos J, Orellana C, Sanmarti R, et al. Secondary amyloidosis in ankylosing spondylitis. A systematic survey of 137 patients using abdominal fat aspiration. J Rheumatol 1997; 24: 912-915.

6. Tublin ME, Bude RO, Platt JF. The Resistive index in renal Doppler sonography: where do we stand? AJR Am J Roentgenol 2003; 180: 885-892.

7. Malatino LS, Polizzi G, Garozzo M, et al. Diagnosis of renovascular disease by extra- and intrarenal Doppler parameters. Angiology 1998; 49: 707-721.

8. Platt JF, Rubin JM, Ellis JH. Lupus nephritis: predictive value of conventional and Doppler US and comparison with serologic and biopsy parameters. Radiology 1997; 203: 82-86.
9. Khan MA. Update on spondyloarthropathies. Ann Intern Med 2002; 136: 896-907.

10. Zeboulon N, Dougados M, Gossec L. Prevalence and characteristics of uveitis inspondylarthropathies: a systematic literature review. Ann Rheum Dis 2008; 67: 955-959.

11. Resorlu H, Akbal A, Resorlu M, et al. Epicardial adipose tissue thickness in patients with ankylosing spondylitis. Clin Rheumatol 2015; 34: 295-299.

12. Heeneman S, Daemen MJ. Cardiovascular risks in spondyloarthritides. Curr Opin Rheumatol 2007; 19: 358-362.

13. Senocak O, Manisali M, Özaksoy D, Sevinç C, Akalin E. Lung parenchyma changes in ankylosing spondylitis: demonstration with high resolution CT and correlation with disease duration. Eur J Radiol 2003; 45: 117-122.

14. Canales BK, Leonard SM, Singh JA, et al. Spondyloarthropathy: an independent risk factor for kidney stones. J Endourol 2006; 20: 542-546.

15. Vilar MJ, Cury SE, Ferraz MB, Sesso R, Atra E. Renal abnormalities in ankylosing spondylitis. Scand J Rheumatol 1997; 26: 19-23.

16. Dönmez S, Pamuk ÖN, Pamuk GE, Aydoğdu E, Inman R. Secondary amyloidosis in ankylosing spondylitis. Rheumatol Int 2013; 33: 1725-1729.

17. Singh G, Kumari N, Aggarwal A, Krishnani N, Misra R. Prevalence of subclinical amyloidosis in ankylosing spondylitis. J Rheumatol 2007; 34: 371-373.

18. Fackovcova D, Kristova V, Kriska M. Renal damage induced by the treatment with non-opioid analgesics - theoretical assumption or clinical significance. Bratisl Lek Listy 2000; 101; 8: 417-422.

19. Weir MR. Renal effects of nonselective NSAIDs and coxibs. Cleve Clin J Med 2002; 69: SI53-SI58.

20. Platt JF, Ellis JH, Rubin JM, DiPietro MA, Sedman AB. Intrarenal arterial Doppler sonography in patients with nonobstructive renal disease: correlation of resistive index with biopsy findings. AJR Am J Roentgenol 1990; 154: 12231227.

21. Platt JF, Rubin JM, Ellis JH. Lupus nephritis: predictive value of conventional and Doppler US and comparison with serologic and biopsy parameters. Radiology 1997; 203: 82- 86 .

22. Aikimbaev KS, Canataroglu A, Ozbek S, Usal A. Renal vascular resistance in progressive systemic sclerosis: evaluation with duplex Doppler ultrasound. Angiology 2001; 52: 697-701.

23. Alterini B, Mori F, Terzani E, et al. Renal resistive index and left ventricular hypertrophy in essential hypertension. Ann Ital Med Int 1996; 11: 107-113.

24. Cece H, Yazgan P, Karakas E, et al. Carotid intima-media thickness and paraoxonase activity in patients with ankylosing spondylitis. Clin Invest Med 2011; 34: 225-231. 